\title{
Eye image effect in the context of pedestrian safety: a French
}

\section{questionnaire study [version 1; peer review: 1 approved with}

\section{reservations]}

\author{
Cédric Sueur (DiD1,2, Anthony Piermattéo33, Marie Pelé ${ }^{3}$ \\ ${ }^{1}$ IPHC, UMR7178, Université de Strasbourg, CNRS, Strasbourg, France \\ ${ }^{2}$ Institut Universitaire de France, Paris, China \\ ${ }^{3}$ ETHICS EA7446, Lille Catholic University, Lille, France
}

\author{
V1 First published: 23 Feb 2022, 11:218 \\ https://doi.org/10.12688/f1000research.76062.1 \\ Latest published: 23 Feb 2022, 11:218 \\ https://doi.org/10.12688/f1000research.76062.1
}

\begin{abstract}
Human behavior is influenced by the presence of others, which scientists also call 'the audience effect'. The use of social control to produce more cooperative behaviors may positively influence road use and safety. This study uses an online questionnaire to test how eyes images affect the behavior of pedestrians when crossing a road. Different eyes images of men, women and a child with different facial expressions -neutral, friendly and angry- were presented to participants who were asked what they would feel by looking at these images before crossing a signalized road. Participants completed a questionnaire of 20 questions about pedestrian behaviors (PBQ). The questionnaire was received by 1,447 French participants, 610 of whom answered the entire questionnaire. Seventy-one percent of participants were women, and the mean age was $35 \pm 14$ years. Eye images give individuals the feeling they are being observed at $33 \%$, feared at $5 \%$ and surprised at $26 \%$, and thus seem to indicate mixed results about avoiding crossing at the red light. The expressions shown in the eyes are also an important factor: feelings of being observed increased by about 10-15\% whilst feelings of being scared or inhibited increased by about $5 \%$ as the expression changed from neutral to friendly to angry. No link was found between the results of our questionnaire and those of the Pedestrian Behavior Questionnaire (PBQ). This study shows that the use of eye images could reduce illegal crossings by pedestrians, and is thus of key interest as a practical road safety tool. However, the effect is limited and how to increase this nudge effect needs further consideration.
\end{abstract}

\section{Keywords}

prosociality, road crossing, reputation, accident prevention, pedestrian behavior

\section{Open Peer Review \\ Approval Status? \\ 1 \\ version 1

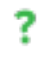 \\ 23 Feb 2022 \\ view \\ 1. Yunchang Zhang, Desay SV, Singapore, \\ Singapore \\ Any reports and responses or comments on the article can be found at the end of the article.}


Corresponding author: Cédric Sueur (cedric.sueur@iphc.cnrs.fr)

Author roles: Sueur C: Conceptualization, Data Curation, Formal Analysis, Funding Acquisition, Investigation, Methodology, Project Administration, Software, Supervision, Validation, Visualization, Writing - Original Draft Preparation; Piermattéo A: Investigation, Methodology, Validation, Writing - Review \& Editing; Pelé M: Conceptualization, Formal Analysis, Funding Acquisition, Investigation, Methodology, Project Administration, Supervision, Validation, Writing - Review \& Editing

Competing interests: No competing interests were disclosed.

Grant information: The author(s) declared that no grants were involved in supporting this work.

Copyright: $(2022$ Sueur $C$ et al. This is an open access article distributed under the terms of the Creative Commons Attribution License, which permits unrestricted use, distribution, and reproduction in any medium, provided the original work is properly cited.

How to cite this article: Sueur C, Piermattéo A and Pelé M. Eye image effect in the context of pedestrian safety: a French questionnaire study [version 1; peer review: 1 approved with reservations] F1000Research 2022, 11:218

https://doi.org/10.12688/f1000research.76062.1

First published: 23 Feb 2022, 11:218 https://doi.org/10.12688/f1000research.76062.1 


\section{Introduction}

Whatever the size and complexity of the society we live in, social life involves respecting rules or norms in order to maintain peace and cohesion (Coleman, 1994; Elster, 1989; Fehr and Fischbacher, 2004). Violating these social norms can unbalance the public good insofar that law-breakers will gain more benefits than their honest counterparts, or other individuals will be put at risk. In order to balance costs and benefits, punishment or police behaviors have evolved in humans societies (Fehr and Gächter, 2000, 2002), and in other primate societies (Boyd and Richerson, 1992; Flack et al., 2006; Mendes et al., 2018; Riedl et al., 2012). Like other primate species, humans have developed emotional bases for prosocial behaviors allowing cooperation. These emotions are concerns, empathy and a sense of morality and of reputation (De Waal, 2006; Jensen et al., 2014; Keltner and Anderson, 2000; Penner et al., 2005; Tomasello and Vaish, 2013) and they are defined as moral emotions by Haidt (2003). Being concerned or empathic enables humans to recognize when they are doing something wrong and correct their behavior in order to maintain a prosocial reputation and continue interacting cooperatively with their conspecifics (Alexander, 1987; Bateson et al., 2006; Burnham and Johnson, 2005).

Our behavior is therefore influenced by the presence of others, which scientists also call 'the audience effect' (Filiz-Ozbay and Ozbay, 2014; Kurzban et al., 2007; Zuberbühler, 2008). Feeling observed by real persons or an imagined audience, therefore has an impact on human behavior. Embarrassment, for instance, is defined as the 'acute state of flustered, awkward, abashed chagrin that follows events that increase the threat of unwanted evaluations from real or imagined audiences' (Eller et al., 2011; Miller, 1996). Being observed also tends to make individuals more compliant (Dear et al., 2019; Rodriguez Mosquera et al., 2011). With this in mind, Bateson et al. (2006) conducted an experiment to test the effect of eye images on cooperative behavior. In the coffee area of a building at the University of Newcastle, an honesty box was used for people to make contributions to the coffee fund. The experiment consisted of placing pictures of eyes or flowers close to this box and assessing whether they led to differences in the contributions made. The authors found that in the presence of eye images, subjects paid on average 2.76 times more than when flowers images were displayed. Still comparing the effect of these flowers images as control to those of eye images, other studies have found similar prosocial effects during everyday events. For example, eye images had prosocial effects such as cooperation for the clearing of trays in a university cafeteria (Ernest-Jones et al., 2011) and for waste sorting at a bus stop (Francey and Bergmüller, 2012). Similar results were found in experiments in more specific contexts such as blood donations: While eye images on flyers did not result in differences in pledge with a logo as control, more "real" donations were made by students who got the flyers with eyes image (Sénémeaud et al., 2017). Other than the activation of a "sense of being seen" (Pfattheicher and Keller, 2015) or the desire to maintain a pro-social reputation (Bateson et al., 2006), it is important to note that humans possess neurons that respond to faces and eyes and activate such prosocial behaviors (Emery, 2000; Haxby et al., 2000). Bateson et al. (2013) suggest that eye images induce more pro-social behavior regardless of local norms, thus suggesting that the application of eye images could be a means to combat anti-social behavior by triggering a feeling of shame (Nugier et al., 2007).

The use of social control to produce more cooperative behaviors may positively influence road use and safety. The limitation of conflicts and accidents on road infrastructures is directly dependent upon the respecting of rules by the numerous pedestrians and drivers. However, more than 8000 pedestrians die in road accidents in Europe every year, $25 \%$ of whom die when using crosswalks (Guéguen et al., 2015, 2016). These lethal accidents are due to cars not stopping at signalized intersections but also to pedestrians crossing illegally at the red signal (Sueur et al., 2013). Past studies show that individuals do not cross illegally when other pedestrians are present (Pelé et al., 2017, 2019a, 2019b). Visual communication between two individuals can lead to a change in the receiver's behavior and this effect can be found in a road context. For example, eye contact is an important element. Some studies explored the effect of gaze and smile on the propensity of drivers to stop at signalized intersections and allow pedestrians to cross (Guéguen et al., 2015, 2016). A pedestrian waiting at the edge of an unmarked crosswalk has a greater likelihood to cross if s/he seeks to make visual contact with an approaching driver than if s/he is not looking towards the approaching car, with $67.7 \%$ of cars stopping versus $55.1 \%$, respectively (Guéguen et al., 2015). If in addition to this visual contact the pedestrian smiles, $62.9 \%$ of drivers stopped compared to $50.1 \%$ if the pedestrian sought visual contact with a neutral face (Guéguen et al., 2016). These studies show that visual contact can modify the behavior and speed of drivers, and highlight that the facial expression of the pedestrian also has an impact (Ren et al., 2016). However, these studies are rare and more research is needed on how human facial expressions affect the probability that pedestrians will cross the road illegally. This research may have great potential in terms of applications in the field of road safety, especially regarding the regulation of pedestrian behaviors.

This study aims to test the effect of eye images on the behaviors of pedestrians crossing at the red light. We collected different images of eyes from five different persons (two men, two women and one child) expressing different facial expressions (neutral, friendly and angry) and one image of flowers for use as a control (Figure 1). These images were used in an online questionnaire that asked participants what they would feel if they saw these images before crossing a road. 


\section{Control}

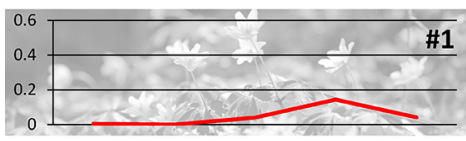

\section{Eyes images}

\section{Friendly}
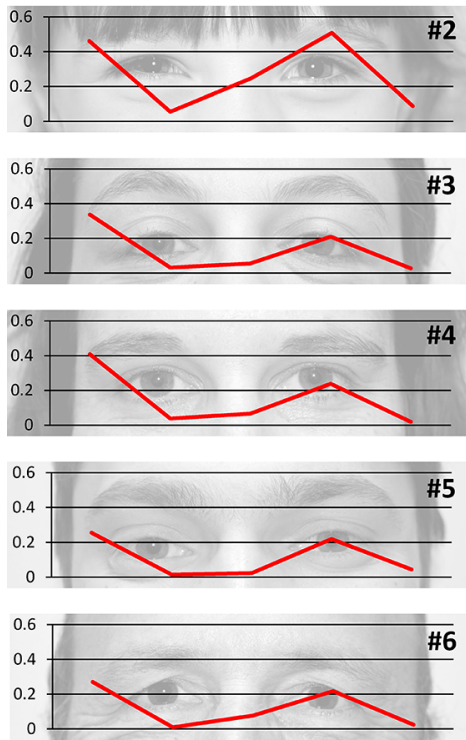

Neutral
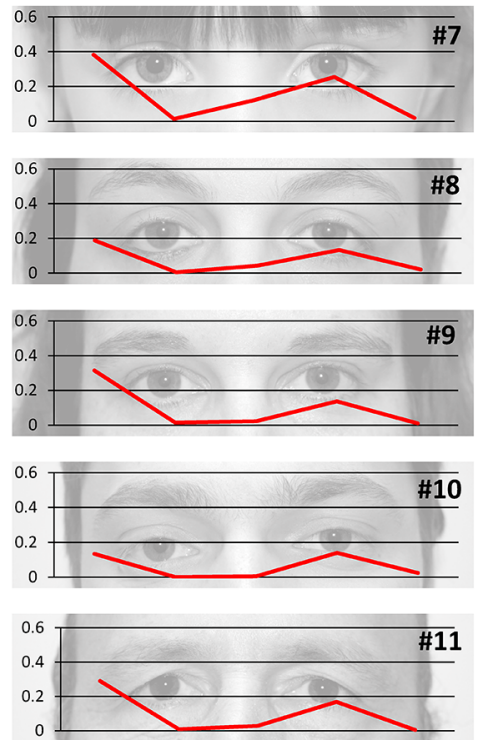

Legend \& scale

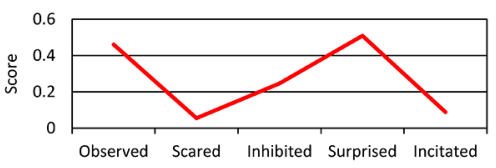

Angry
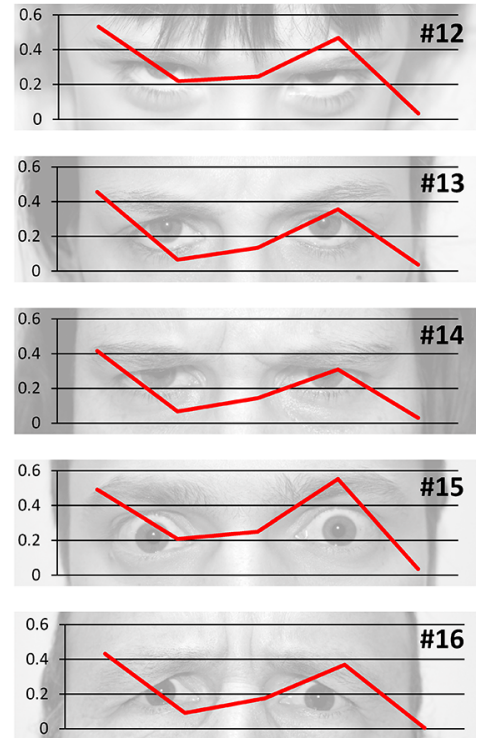

Figure 1. Images used to understand the effect of eye images on pedestrian behaviors. The flower image is a control. The effect of eye images was tested using three different expressions (friendly, neutral and angry respectively from left to right) in the eyes of five different persons. Five questions were asked about feelings for each eye image: Do you feel observed, scared, inhibited, surprised or encouraged? (See methods for details). The score for each feeling and each image is the average score for all participants. Image credits: Cédric Sueur.

Would they feel observed, scared or surprised? Would the images discourage or encourage them to cross at the red light? These questions were chosen with caution in order to make the questionnaire valid and are based on previous studies (Bateson et al., 2013; Ernest-Jones et al., 2011; Francey and Bergmüller, 2012; Saeed et al., 2020). We compared their answers to these questions with sociodemographic variables (gender, age, geographical zone and city size) and also with a previous well-known questionnaire called 'Pedestrian Behavior Questionnaire' (hereafter referred to as PBQ, Appendix A: Deb et al., 2017; Granié et al., 2013), which tested the propensity of pedestrians to violate rules, make errors or lapses, or show positive or aggressive behaviors. The angry eye images are expected to have a stronger emotional impact on participants and thus prevent them from crossing illegally (Bateson et al., 2006). We also expect a gender effect and an age effect, with a stronger impact of eye images on women and younger individuals. Indeed, women might feel more observed or scared than men when looking at the eye images; studies have shown women to be more empathic and thus more receptive in different situations (Flynn, 2000; Furnham et al., 2003; Kellert \& Berry, 1987; Miller et al., 2009). In the context of road crossing, fewer illegal and risky behaviors are observed in women than in men (Holland and Hill, 2007; Pelé et al., 2017; Sueur et al., 2013; Tom and Granié, 2011), with more positive behaviors and fewer rule violations or aggressive behaviors (Deb et al., 2017; Tom and Granié, 2011). Studies show that younger people are less respectful of rules (Holland and Hill, 2007; Pelé et al., 2017; Pfeffer and Hunter, 2013). We also expect regional differences in reactions to eye images, as interregional differences were observed in road accidents (Eksler et al., 2008; Lassarre and Thomas, 2005). Correlations are expected between the responses to the eye image questionnaire and the PBQ. Indeed, participants showing higher scores for violations or aggressive behaviors in the PBQ (i.e. the less prosocial individuals) should be less affected by eye images than participants who show more positive behaviors (i.e., the more empathic and cooperative individuals).

\section{Methods}

\subsection{Questionnaire}

The questionnaire was designed in three steps: 1. Eye images, 2. Pedestrian behavior questionnaire and 3. Sociodemographic questions. 
Eye images: To test the effect of eye images, pictures were taken of five different individuals (one child, two women and two men) with three different facial expressions (friendly, neutral and angry). A picture of flowers was used as a control. Each picture was taken in black and white with the same contrast and brightness calibration (see Figure 1). For each of the 16 pictures (15 eyes and 1 control), participants were asked to answer five questions about their feelings when looking at the picture:

1. Observation: Do you feel observed when looking at this picture? Answer: yes/maybe/no

2. Fear: Are you scared when looking at this picture? Answer: yes/maybe/no

3. Inhibition: Would looking at this picture prevent you from crossing at the red light? Answer: yes/maybe/no

4. Surprise: Would this picture make you feel surprised if you saw it before crossing the road? Answer: yes/maybe/no

5. Incitation: Would seeing this picture encourage you to cross at the red pedestrian signal? Answer: yes/maybe/no

These five questions were chosen with caution. In order to check the validity of the questionnaire, we chose the four first questions to evaluate the negative effects of the eyes images and the fifth question to evaluate a positive effect. This means that we expected to have the scores of the fourth first questions to be positively correlated between them but negatively correlated with the fifth question. The questionnaire was validated with results (Section 3.1.) following our predictions.

As five questions for 16 pictures results in a very long questionnaire of 80 questions, 4 images (with for each image the five questions) were randomly selected for the participants to answer. We checked for a homogeneous distribution of questions to participants by making packages of 20 questions (for 4 images).

Sociodemographic questions: Participants were asked to indicate their gender (female or male), age (as numeric) and French postal code. The French postal code provides the city population size (DataNova Opensource, 2014 census) and the Defense and Security Zones (French Homeland Ministrysee https://doi.org/10.5281/zenodo.5745446.

Pedestrian behavior questionnaire $(P B Q)$ : Participants completed a questionnaire of 20 questions about pedestrian behaviors by Deb et al. (2017). We used a pedestrian behavior questionnaire (PBQ) with 20 questions, developed by (Deb et al., 2017) and taken from (Tom and Granié, 2011). The order of questions was randomly attributed. In parenthesis, the average $\pm \operatorname{stdv}$ of the score (from 1 - never - to 6 - always -) attributed by participants to each question $(\mathrm{N}=611)$.

\section{Violations (V)}

V1 I cross the street even though the pedestrian light is red. $(3.73 \pm 1.35)$

V2 I cross diagonally to save time. $(3.45 \pm 1.42)$

V3 I cross outside the pedestrian crossing even if there is one (crosswalk) less than $50 \mathrm{~m}$ away. (3.51 \pm 1.49$)$

V4 I take passageways forbidden to pedestrians to save time. $(2.35 \pm 1.36)$

\section{Errors (E)}

E1 I cross between vehicles stopped on the roadway in traffic jams. (3.34 \pm 1.43$)$

E2 I cross even if vehicles are coming because I think they will stop for me. (2.36 \pm 1.19$)$

E3 I walk on cycling paths when I could walk on the sidewalk. $(1.91 \pm 0.96)$

E4 I run across the street without looking because I am in a hurry. $(1.27 \pm 0.63)$ 


\section{Lapses (L)}

L1 I realize that I have crossed several streets and intersections without paying attention to traffic. $(1.52 \pm 0.82)$

L2 I forget to look before crossing because I am thinking about something else. (1.65 \pm 0.78$)$

L3 I cross without looking because I am talking with someone. (1.65 \pm 0.85$)$

L4 I forget to look before crossing because I want to join someone on the sidewalk on the other side. $(1.45 \pm 0.70)$

\section{Aggressive Behaviors (A)}

A1 I get angry with another road user (pedestrian, driver, cyclist, etc.), and I yell at him. (1.94 \pm 1.17$)$

A2 I cross very slowly to annoy a driver. $(1.45 \pm 0.87)$

A3 I get angry with another road user (pedestrian, driver, cyclist, etc.), and I make a hand gesture. (2.08 \pm 1.20$)$

A4 I have gotten angry with a driver and hit their vehicle. $(1.25 \pm 0.70)$

\section{Positive Behaviors (P, Reverse-scaled items)}

P1 I thank a driver who stops to let me cross. $(5.55 \pm 0.82)$

P2 When I am accompanied by other pedestrians, I walk in single file on narrow sidewalks so as not to bother the pedestrians I meet. $4.05 \pm 1.46$ )

P3 I walk on the right-hand side of the sidewalk so as not to bother the pedestrians I meet. (4.04 \pm 1.48$)$

P4 I let a car go by, even if I have the right of way, if there is no other vehicle behind it. (3.60 \pm 1.49$)$

Questions were presented in random order. PBQ was the first complete questionnaire to study a broad range of aspects of pedestrian behavior on the road for all age groups. This questionnaire was originally developed by Tom and Granié (2011) with 47 questions. We chose the short version, which is considered as reliable as the long version according to Deb et al. (2017) and Tom and Granié (2011), in order to avoid demotivating participants. The 20 questions are categorized into five items as followed:

1. Transgression: deliberate deviation from social rules without intention to cause injury or damage, Reason et al., 1990;

2. Error: deficiency in knowledge of traffic rules and/or in the inferential processes involved in making a decision, Rasmussen, 1980; Reason et al., 1990;

3. Lapse: unintentional deviation from practices related to a lack of concentration on the task; forgetfulness, Reason et al., 1990;

4. Aggressive Behavior: tendency to misinterpret other road users' behavior, resulting in the intention to annoy or endanger, Baxter et al., 1990; Lawton et al., 1997;

5. Positive Behavior: behavior that seeks to avoid violation or error and/or seeks to ensure traffic rule compliance, Özkan and Lajunen, 2005.

The participants were required to answer the questions using a 6-point Likert scale (1-very infrequently or never, 2-quite infrequently, 3-infrequently, 4-frequently, 5-quite frequently, 6-very often or always). 


\subsection{Survey administration and participants}

The survey was created using LimeSurvey (Engard, 2009; Jayasundara et al., 2010; LimeSurvey Project Team, 2012) and administered online to the French population through mails and social media (Facebook and Twitter).

The questionnaire was received by 1,447 participants, 610 of whom answered the entire questionnaire (all three steps). The resulting dataset was used in our analyses $(\mathrm{N}=610)$. The mean time to answer the entire questionnaire was seven minutes. $71 \%$ of participants were women, and the mean age was $35 \pm 14$ years. The geographic repartition of the population is as follows: Hauts-de-France $(\mathrm{N}=17)$, Ile-de-France $(\mathrm{N}=100)$, Ouest $(\mathrm{N}=92)$, Est ( $\mathrm{N}=219)$, Sud-Ouest $(\mathrm{N}=18)$, Sud-Est $(\mathrm{N}=75)$, Sud $(\mathrm{N}=89)$. These factors were included in statistical analyses to avoid selection biases selection.

\subsection{Research ethics}

All data were anonymous, and participants were given sequential numerical identities corresponding to the moment they answered the questionnaire. Participants could obtain information about the study and its results by contacting the authors via an email address provided at the end of the questionnaire. We followed the ethical guidelines of our institution (CNRS-IPHC, Strasbourg, France). This study received ethical approval from the road security direction of the French Homeland Ministry (RefCNRS190529).

Formal written agreement or parental consent was obtained from the five people photographed for the eye images. They were aware of, and agreed to, the intended use of the photographs in the questionnaire and in the publication.

\subsection{Statistical analyses}

We first calculated the mean score for each question and each image. This score ranges from 0 to 1 , where 0 indicates $100 \%$ of participants answering "No" to the question and 1 corresponds to $100 \%$ answering "Yes". The scores concerning all three answers [Yes, maybe, No] and the scores concerning only two answers [Yes, No] are correlated at 95\% (linear regression, $\mathrm{P}<0.0001, \mathrm{R}^{2}=0.95, \mathrm{~N}=80$ ). For this reason and in order to simplify statistical analyses, we only used the [Yes, No] answers.

A Pearson correlation test was used to check the correlation between each feeling. We then performed a principal component analysis (PCA), followed by Hierarchical Clustering on Principal Components (HCPC) in order to assess which images resulted in higher scores. Following these analyses, the incitation question was excluded from the next statistical tests (see results) and a mean score was calculated for each participant, combining all four remaining questions.

We assessed whether this score is influenced by the age, gender, geographical zone of their city or the city population size (categorized according to quartiles). A general linear model (glm) with a normal law was used with the R package "MultComp" for multiple comparisons (Bretz et al., 2016). A separate GLM tested the interactions between age/sex factors and the expression of eye images (i.e., neutral, angry, friendly). The conditions of application (normality and homoscedasticity of residuals) were graphically verified.

The 20 PBQ questions with values from 1 to 6 were analyzed using a PCA with a varimax rotation (Package R Psych; Revelle, 2011; Revelle \& Revelle, 2015), following the procedure explained in Granié et al. (2011, 2013). In order to fit with the PCA axes of these studies, we set a maximum number of four loadings (as a preliminary analysis of five PCA dimensions shows a division of the positive behaviors in dimensions 4 and 5, we combined both dimensions as described by Granié et al., 2011, 2013). The coordinates of participants in each dimension (five with loadings higher than 1.00) were then compared with the eye images mean score using a Pearson correlation test. GLM analysis was also carried out to test coordinates with gender, age and city data of participants using. The four dimensions were scaled and normalized using the scale function in R. For the "zone" variable, an Anova followed by a Tukey posthoc test was performed on the GLM residuals.

All tests were carried out with R 3.6 (R Development Core Team, 2009). The significance level was set at 0.05. Results are indicated with mean \pm stdv

\section{Results}

\subsection{What do participants feel when seeing the eye images?}

Whatever the eye image, the mean score for the 'Observation' question is $0.33 \pm 0.14$ (meaning that $33 \%$ of participants answered 'Yes' to this question and therefore feel observed). The mean score for the 'Fear' question is $0.05 \pm 0.07$. The mean score for the 'Inhibition' question is $0.10 \pm 0.08$. The mean score for the 'Surprise' question is $0.26 \pm 0.12$. Finally, the mean score for the 'Incitation' question is $0.02 \pm 0.01$. Scores for each image and each question are provided in Table 1 and are shown in Figure 1. 
Table 1. Mean score for each eye image (except the flower control image) and each feeling-related question. Images are ranked according to their values in dimension 1 of the PCA, without the incitation variable. Colors indicate the clusters assessed by the HCPC, from the least intense (green) to the most intense (red) feeling.

\begin{tabular}{|l|l|l|l|l|l|l|l|}
\hline Category & $\begin{array}{l}\text { Facial } \\
\text { expression }\end{array}$ & Observation & Fear & Inhibition & Surprise & Incitation & $\begin{array}{l}\text { PCA Dim1 } \\
\text { Coord }\end{array}$ \\
\hline Flower & - & 0.00 & 0.00 & 0.04 & 0.14 & 0.04 & -2.45 \\
\hline Man & Neutral & 0.13 & 0.00 & 0.00 & 0.14 & 0.02 & -2.25 \\
\hline Woman & Neutral & 0.19 & 0.00 & 0.04 & 0.13 & 0.02 & -1.80 \\
\hline Woman & Neutral & 0.31 & 0.01 & 0.02 & 0.14 & 0.01 & -1.41 \\
\hline Man & Neutral & 0.29 & 0.01 & 0.03 & 0.17 & 0.00 & -1.37 \\
\hline Man & Friendly & 0.26 & 0.02 & 0.02 & 0.22 & 0.04 & -1.29 \\
\hline Man & Friendly & 0.27 & 0.01 & 0.08 & 0.22 & 0.02 & -0.95 \\
\hline Woman & Friendly & 0.34 & 0.03 & 0.06 & 0.21 & 0.03 & -0.72 \\
\hline Woman & Friendly & 0.41 & 0.04 & 0.07 & 0.24 & 0.02 & -0.25 \\
\hline Child & Neutral & 0.38 & 0.01 & 0.12 & 0.25 & 0.02 & -0.10 \\
\hline Woman & Angry & 0.42 & 0.07 & 0.14 & 0.31 & 0.03 & 0.75 \\
\hline Woman & Angry & 0.46 & 0.07 & 0.13 & 0.36 & 0.04 & 1.01 \\
\hline Man & Angry & 0.43 & 0.09 & 0.18 & 0.37 & 0.00 & 1.41 \\
\hline Child & Friendly & 0.46 & 0.06 & 0.25 & 0.51 & 0.09 & 2.24 \\
\hline Child & Angry & 0.53 & 0.22 & 0.25 & 0.47 & 0.03 & 3.52 \\
\hline Man & Angry & 0.49 & 0.21 & 0.25 & 0.55 & 0.04 & 3.66 \\
\hline
\end{tabular}

Pearson's correlation tests between scores (Figure 2) showed a high correlation between all feelings $(\mathrm{r}>0.8, \mathrm{p}<0.001)$ excluding incitation $(\mathrm{r}<0.39, \mathrm{p}>0.05)$. Indeed, a PCA showed that dimension 1 was composed of four feelings (Observation, Fear, Inhibition and Surprise, $\mathrm{r}>0.85 \mathrm{see}$ https://doi.org/10.5281/zenodo.5745446 for details) and explained $72.73 \%$ of variance in the scores, whilst dimension 2 , which was solely composed of the Incitation feeling $(\mathrm{r}=0.89)$, explained $19.28 \%$ of variance. These results validate our questionnaire with participants answering coherently following our predictions. A HCPC following this PCA produced five clusters, without including picture 2 (Child's friendly eyes). When the incitation question was removed from analyses, dimension 1 ( $\mathrm{r}>0.88$ ) explained $87.06 \%$ of score variance whilst dimension $2(\mathrm{r}<0.45)$ explained only $7.28 \%$. The resulting HCPC produced four clusters, with picture 2 regrouped with another cluster. In view of these results and the aims of our study, we decided to discard the incitation question for the following analyses. The images were then grouped into four clusters. The first cluster includes the flower, and a man and a woman with neutral expressions. It has a lower influence on the scores (see green elements in Table 1; participants do not feel surprised, scared, observed or inhibited). The fourth and last cluster (see red elements in Table 1) is composed of the child and a man with an angry expression. This cluster shows the highest scores (i.e. participants felt observed, scared, surprised and inhibited). Gender is therefore equally distributed in these four clusters, but the picture of a child's eyes has a strong effect on feelings (ranked 10, 14 and 15 on the 16 images, Table 1). Moreover, whilst the first cluster includes only 'neutral' images and the second cluster includes 'friendly' and 'neutral' images, all the angry images are found in the third and fourth clusters, meaning that these expressions lead to stronger feelings.

\subsection{How did the influence of eye images on the feelings of participants vary according to their sociodemographic factors?}

The mean score for each participant was influenced by gender and age (Figure 3) but not by the geographical zone or the city population size (see Table 2 for statistical values). Men have a lower score than women, meaning that they feel less scared or observed by eyes. Age negatively influences the score, meaning that older participants have a lower score and feel less observed or scared than youngers. When we tested the effect on the mean score of the interactions of age and sex with the eye expressions (i.e., neutral, angry or friendly), we did not found any interaction between age and eye expression ( $\mid \mathrm{t}-\mathrm{valuel}<1.086, \mathrm{p}>0.277$ ) or between sex and eye expression (It-valuel< $1.449, \mathrm{p}>0.147$ ).

\subsection{Answers of participants to the pedestrian behavior questionnaire}

The 20 PBQ questions were analyzed in the same way as those in referenced studies (Deb et al., 2017; Granié et al., 2013; Tom and Granié, 2011). Results are in accordance with the precited studies (see https://doi.org/10.5281/zenodo.5745446 


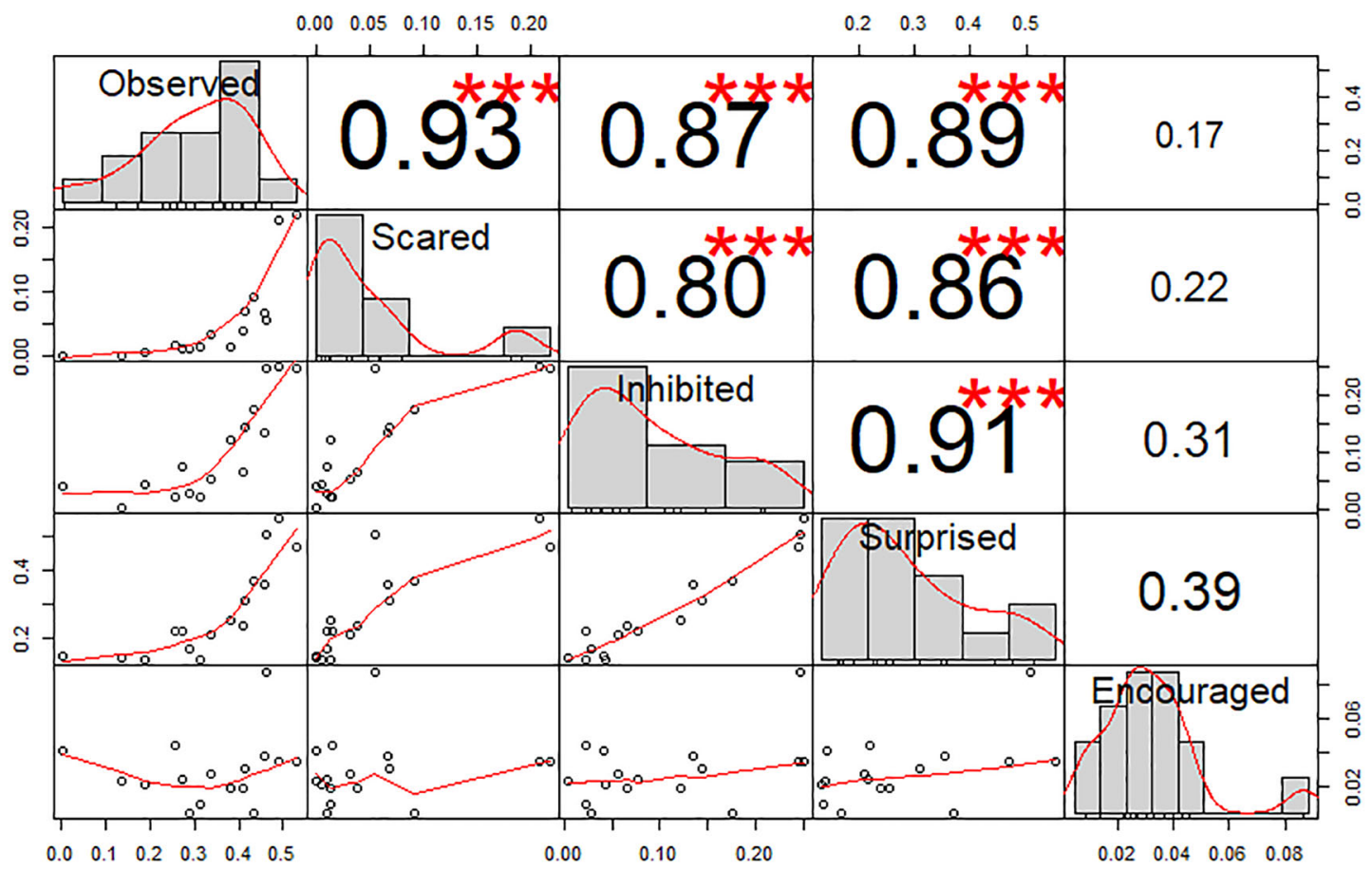

Figure 2. Chart of correlations between the feelings scores. Values indicate the Pearson correlation. Stars indicate statistical significance (Absence $=p>0.05 ; * \star *=p<0.001$ ).
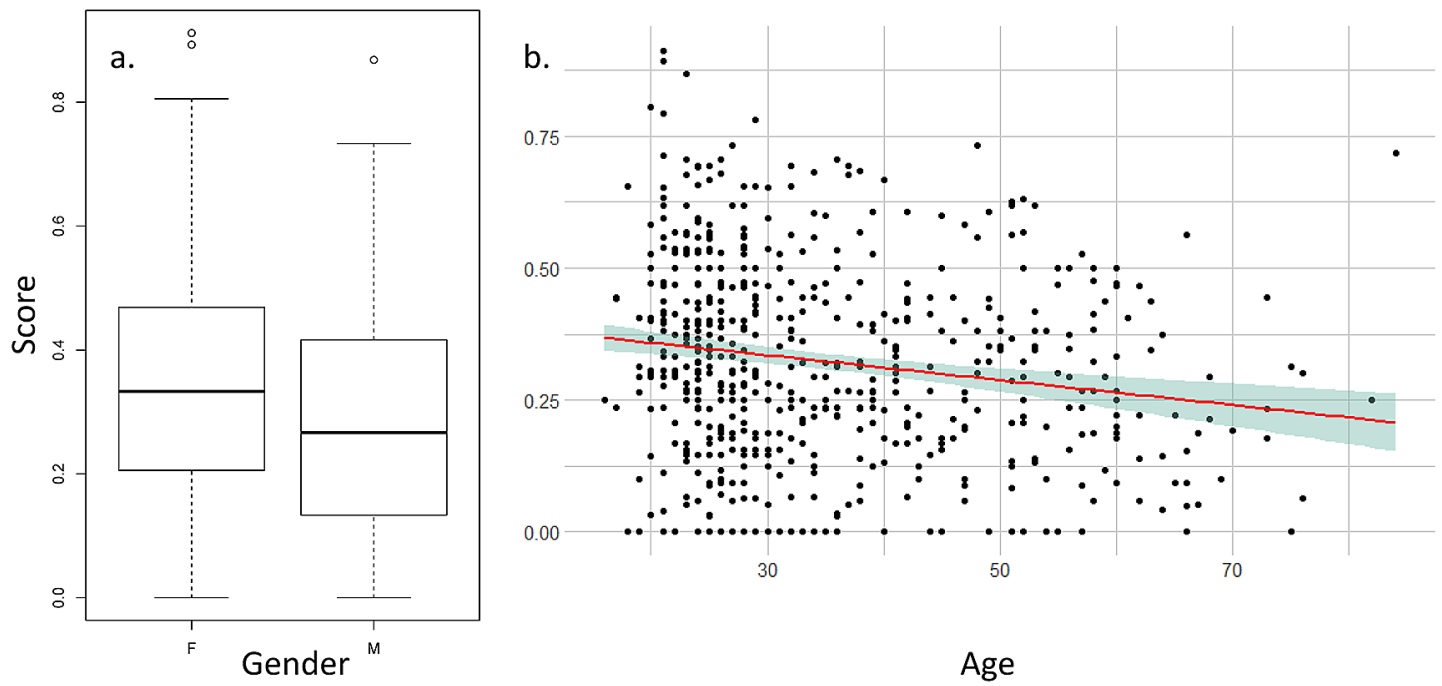

Figure 3. Influence of gender (a.) and age (b.) on the mean score of the feelings of participants on seeing the eye images.

for details). The results explain $49.7 \%$ of total variance. The Kaiser-Meyer-Olkin measure of sampling adequacy was satisfactory $(0.80)$ and the Bartlett's test of sphericity was significant $(\mathrm{p}<0.0001)$. The first dimension explains $15.7 \%$ of variance and corresponds to "lapses" (following a loading above 0.4 ). The second dimension explained $14.7 \%$ of variance and corresponds to "transgressions", meaning violations and errors. The third dimension explains $11.2 \%$ of variance and corresponds to "aggressive behaviors". Finally, the fourth dimension explains $8.2 \%$ of variance and corresponds to "positive behaviors". 
Table 2. Statistical values for the general linear model with the mean score of the eye images questionnaire. HDF for Hauts-de-France, IDF for Ile-de-France.

\begin{tabular}{|l|l|l|l|l|}
\hline & Estimate & Std. Error & $\mathbf{z}$ value & $\operatorname{Pr}(>|\mathbf{z}|)$ \\
\hline (Intercept) & 0.4071361 & 0.0306555 & 13.281 & $<0.001$ \\
\hline Gender [Male] & -0.050761 & 0.0164812 & -3.08 & 0.0198 \\
\hline Age & -0.0022274 & 0.0005603 & -3.975 & $<0.001$ \\
\hline ZoneHDF & 0.0089592 & 0.0461934 & 0.194 & 1 \\
\hline ZoneIDF & 0.0398962 & 0.0221828 & 1.799 & 0.4841 \\
\hline ZoneOuest & 0.0538668 & 0.023243 & 2.318 & 0.172 \\
\hline ZoneSud & 0.0259914 & 0.02306 & 1.127 & 0.9272 \\
\hline ZoneSudEst & -0.0156868 & 0.0246333 & -0.637 & 0.9986 \\
\hline ZoneSudOuest & -0.0018212 & 0.0449061 & -0.041 & 1 \\
\hline Population size & -0.0030641 & 0.0066881 & -0.458 & 0.9999 \\
\hline
\end{tabular}

3.4 How did the PBS axes influence participants according to their sociodemographic factors?

All statistical results are indicated in Table 3. Posthoc Tukey multiple comparisons are detailed in https://doi.org/10.5281/ zenodo.5745446. The "lapses" dimension is only influenced by the city population size, with a larger city population size leading to higher occurrence of unintentional deviation from rules. The "transgressions" dimension (violations and errors) is influenced by age, with younger people making more transgressions, and also by geographical zones, with a higher transgressions score for the "Sud" (South) Zone than the "Est" (East) area (however, see the Tukey test for further details). The "aggressive behaviors" axis is influenced only by the zone but the posthoc test revealed no differences. Finally, the positive behaviors dimension is influenced by gender, with women showing more positive behavior than men do.

Table 3. Statistical values of the general linear models concerning the PBQ dimensions. HDF for Hauts-deFrance, IDF for Ile-de-France. QuartilePop indicates size of towns populations as quartiles.

\begin{tabular}{|c|c|c|c|c|}
\hline & Estimate & Std. Error & t value & $\operatorname{Pr}(>|t|)$ \\
\hline \multicolumn{5}{|l|}{ Lapses dim. } \\
\hline (Intercept) & 0.9604247 & 0.027685 & 34.691 & $<2 \mathrm{e}-16$ \\
\hline Gender [Male] & -0.0289539 & 0.0177072 & -1.635 & 0.1025 \\
\hline Age & -0.0002319 & 0.0003504 & -0.662 & 0.5083 \\
\hline ZoneHDF & -0.0567546 & 0.0495352 & -1.146 & 0.2524 \\
\hline ZoneIDF & -0.0104238 & 0.0238461 & -0.437 & 0.6622 \\
\hline ZoneOuest & 0.0060215 & 0.0249557 & 0.241 & 0.8094 \\
\hline ZoneSud & 0.0128254 & 0.0247557 & 0.518 & 0.6046 \\
\hline ZoneSudEst & -0.0014063 & 0.0264376 & -0.053 & 0.9576 \\
\hline ZoneSudOuest & 0.0219626 & 0.0481632 & 0.456 & 0.6486 \\
\hline QuartilePop & 0.0142764 & 0.0071656 & 1.992 & 0.0468 \\
\hline \multicolumn{5}{|c|}{ Trangressions dim. } \\
\hline (Intercept) & 0.9636292 & 0.0273093 & 35.286 & $<2 \mathrm{e}-16$ \\
\hline Gender [Male] & 0.0299833 & 0.0174669 & 1.717 & 0.08657 \\
\hline Age & -0.0008977 & 0.0003456 & -2.597 & 0.00962 \\
\hline ZoneHDF & 0.0116038 & 0.048863 & 0.237 & 0.81237 \\
\hline ZoneIDF & -0.0023133 & 0.0235225 & -0.098 & 0.92169 \\
\hline
\end{tabular}


Table 3. Continued

\begin{tabular}{|c|c|c|c|c|}
\hline & Estimate & Std. Error & t value & $\operatorname{Pr}(>|t|)$ \\
\hline ZoneOuest & -0.0323359 & 0.0246171 & -1.314 & 0.1895 \\
\hline ZoneSud & 0.0659362 & 0.0244198 & 2.7 & 0.00713 \\
\hline ZoneSudEst & 0.0156855 & 0.0260788 & 0.601 & 0.54776 \\
\hline ZoneSudouest & -0.0083361 & 0.0475096 & -0.175 & 0.86078 \\
\hline QuartilePop & 0.0131719 & 0.0070683 & 1.864 & 0.06288 \\
\hline \multicolumn{5}{|c|}{ Aggressive behav. dim. } \\
\hline (Intercept) & $9.47 \mathrm{E}-01$ & 2.77E-02 & 34.149 & $<2 \mathrm{e}-16$ \\
\hline Gender [Male] & $2.26 \mathrm{E}-02$ & 1.77E-02 & 1.273 & 0.2037 \\
\hline Age & $9.01 \mathrm{E}-05$ & $3.51 \mathrm{E}-04$ & 0.257 & 0.7976 \\
\hline ZoneHDF & $3.28 \mathrm{E}-02$ & 4.96E-02 & 0.661 & 0.5087 \\
\hline ZoneIDF & $1.32 \mathrm{E}-02$ & $2.39 \mathrm{E}-02$ & 0.552 & 0.581 \\
\hline ZoneOuest & $1.88 \mathrm{E}-02$ & $2.50 \mathrm{E}-02$ & 0.752 & 0.4521 \\
\hline ZoneSud & $5.82 \mathrm{E}-02$ & $2.48 \mathrm{E}-02$ & 2.345 & 0.0194 \\
\hline ZoneSudEst & $2.34 \mathrm{E}-02$ & $2.65 \mathrm{E}-02$ & 0.881 & 0.3785 \\
\hline ZoneSudOuest & $3.34 \mathrm{E}-02$ & $4.83 \mathrm{E}-02$ & 0.693 & 0.4888 \\
\hline QuartilePop & $1.69 \mathrm{E}-03$ & $7.18 \mathrm{E}-03$ & 0.235 & 0.814 \\
\hline \multicolumn{5}{|c|}{ Positive behav. dim. } \\
\hline (Intercept) & 1.00474 & 0.0276472 & 36.341 & $<2 \mathrm{e}-16$ \\
\hline Gender [Male] & -0.0399417 & 0.017683 & -2.259 & 0.0243 \\
\hline Age & -0.0005901 & 0.0003499 & -1.686 & 0.0922 \\
\hline ZoneHDF & 0.0104337 & 0.0494676 & 0.211 & 0.833 \\
\hline ZoneIDF & 0.015133 & 0.0238136 & 0.635 & 0.5254 \\
\hline ZoneOuest & 0.0030133 & 0.0249217 & 0.121 & 0.9038 \\
\hline ZoneSud & 0.0129851 & 0.0247219 & 0.525 & 0.5996 \\
\hline ZoneSudEst & -0.0044673 & 0.0264015 & -0.169 & 0.8657 \\
\hline ZoneSudOuest & 0.057924 & 0.0480975 & 1.204 & 0.2289 \\
\hline QuartilePop & 0.0006763 & 0.0071558 & 0.095 & 0.9247 \\
\hline
\end{tabular}

3.5 Link between feelings on seeing the eye images and PBQ axes

We did not identify any statistical correlation between the eye image questionnaire score and the four PBQ dimensions (Figure 4). Lapses dimension: $\mathrm{r}=0.05, \mathrm{t}=1.2843, \mathrm{df}=608$, $\mathrm{p}$-value $=0.1995$; Transgressions dimension: $\mathrm{cor}=0.02$, $\mathrm{t}=0.47498, \mathrm{df}=608, \mathrm{p}$-value $=0.635$, Aggressive behaviors dimension: $\mathrm{r}=-0.07, \mathrm{t}=-1.8156, \mathrm{df}=608, \mathrm{p}$-value $=$ 0.06993; Positive behaviors dimension: $\mathrm{r}=0.05, \mathrm{t}=1.129, \mathrm{df}=608, \mathrm{p}$-value $=0.2593$.

\section{Discussion}

This study tested the potential impact of different eye images - friendly, neutral, and angry -and one image of flowers as a control on pedestrians crossing at the red light. In their responses to an online questionnaire about their feelings on seeing these images, participants revealed that they initially felt observed (about 33\%), then surprised (26\%), then inhibited to cross at the red signal (10\%). Finally, few were scared (5\%) or felt encouraged to cross at the red signal (2\%). Eye images encourage cooperative behavior because unlike pictures of flowers, they make participants feel like they are being watched (Pfattheicher and Keller, 2015). Our results are in line with this explanation, as flowers obtained a null score for being observed or scared. Moreover, the expression conveyed by the eyes also affects participants, as feelings of being observed increased by about 10-15\% whilst feelings of being scared or reluctant to cross the road increased by about $5 \%$ as the expression changed from neutral to friendly to angry. This study confirms that humans react to faces but rates of negative reactions to the eyes are low, indicated a mixed effect. Indeed, we expected more participants to answer that they feel observed, afraid or surprised by the eyes images. Previous studies have shown that this reaction can play a role 

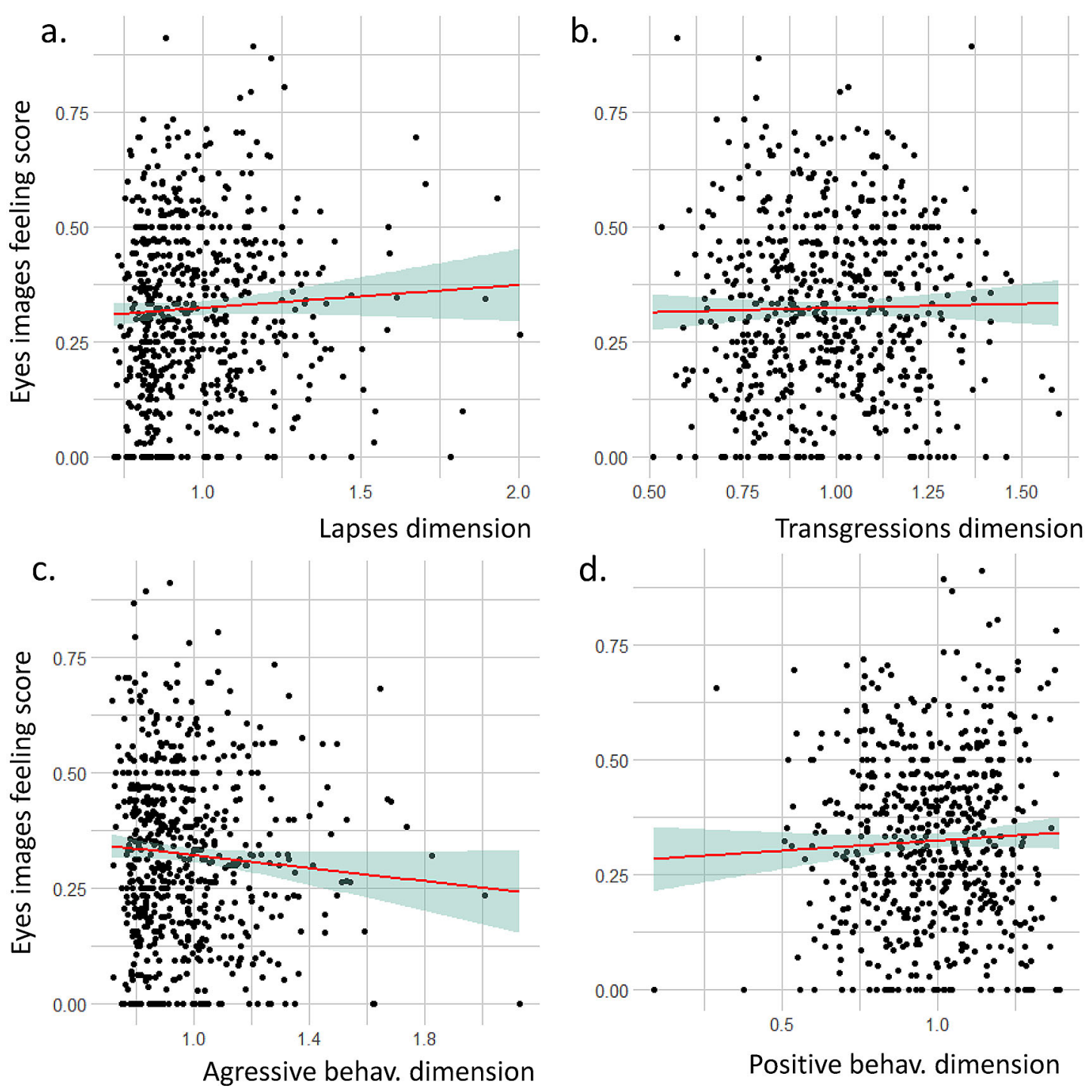

Positive behav. dimension

Figure 4. Relation between the eye image feeling score and the four dimensions of the pedestrian behavior questionnaire (PBQ).

in maintaining the cooperative behaviors that are essential to life in societies (Alexander, 1987; Bateson et al., 2006; Burnham and Johnson, 2005) and it is interesting to put in light our results with these studies.

As predicted by our hypothesis, we found that the gender and age of participants affected their feelings when looking at the eye images. Women were more affected by the images than men were, and younger participants also reacted more than older individuals. Few studies have analyzed the effect of these two variables on the reaction to eye images, maybe because of anonymity in questionnaires or the low number of participants. Two studies report that gender did not influence reactions to eye images (Rodriguez Mosquera et al., 2011; Sparks and Barclay, 2013). However, it is not surprising to find that women felt more observed or scared than men when looking at the eye images, as studies showed women to be more empathic and therefore more receptive in different situations (Flynn, 2000; Furnham et al., 2003; Kellert \& Berry, 1987; Miller et al., 2009). In the context of road crossing, women show also fewer illegal andrisky behaviors than men (Holland and Hill, 2007; Pelé et al., 2017; Sueur et al., 2013; Tom and Granié, 2011). Women also show more positive behaviors, as shown in our study, and fewer violations or aggressive behaviors than men do (Deb et al., 2017; Tom and Granié, 2011).

Although we expected the effect of eye images on feelings to increase with age, the contrary was observed. To our knowledge, this is the first study on the effect of eye images to date that has reported such a result. However, studies of 
pedestrian behaviors showed younger people to be less respectful of rules (Holland and Hill, 2007; Pelé et al., 2017; Pfeffer and Hunter, 2013). This is confirmed in our study, which shows more violations by younger participants than by their older counterparts. Elder pedestrians can also display some illegal behaviors but these are generally due to cognitive or physical disorders (Laurin et al., 2001; Yaffe et al., 2001). Contrary to past studies using PBQ (Deb et al., 2017; Granié et al., 2013), we did not find an age effect on each pedestrian behavior axis. Our results show an effect of the city population size on the lapses dimension, meaning that citizens living in big cities show more unintentional violations and/or are more distracted than the inhabitants of small cities, probably because of the density of the population or visual distractions such as shops, signs or public transport. Participants from the South of France seem to display more violations and aggressive behaviors than those from the rest of France.

No link was found between the results of our questionnaire and those of the Pedestrian Behavior Questionnaire (PBQ). We expected participants showing high scores for violations and aggressive behaviors in the PBQ (or low scores for positive behaviors) to feel less observed or scared by the eye images. However, no correlation was found. This can be explained by a number of reasons. A participant may feel concerned by the eye image but will behave aggressively towards a driver because as a pedestrian, s/he considers that the driver is wrong (and thus seeks to communicate this anger). Alternatively, a participant may not feel concerned by the eyes image because irrespective of who is watching $\mathrm{him} / \mathrm{her}$, s/he will always behave well and consider the behavior of the driver - if the latter also behaves well - as reciprocal (Burnham and Johnson, 2005; Fehr and Fischbacher, 2004). This absence of correlation may be due to differences in the empathic profiles of the population, ranging from people who are naturally cooperative and/or react to eye images in order to ensure they are seen in a positive light, to those who are not cooperative at all and are more likely to react to punishment (Boyd and Richerson, 1992; Fehr and Gachter, 2000). Although we expected people who followed the rules to be nonviolent, the two traits may not be correlated. In other words, a person may react to eye images and be cooperative or follow the rules but be aggressive towards people who do not do likewise. Conversely, another person may be indifferent to others, and will therefore not react to eye images or behave aggressively towards people who do not respect the rules. A second explanation is that the questionnaire is based on a virtual situation that did not affect participants' feelings in the same way as the real situation, thus decreasing the potential correlation between our variables (Francey and Bergmüller, 2012). Past studies have indeed shown a great variability of participants responding to eye images according to the experimental setup that is used (see for instance Fehr \& Schneider, 2010; Northover et al., 2017 for negative results). Anonymity also has a negative impact on the effect of eye images (Lamba and Mace, 2010), and this could have an impact in our study.

\section{Conclusion}

A better understanding of human cooperative behavior in real life is of key interest for social management, from both theoretical and practical perspectives. The present study shows that the use of eye images could help to reduce illegal crossings by pedestrians. However, this effect is limited as not a majority of participants answered that they felt observed, afraid and so on. Drivers react to the smiles and gaze of pedestrians by permitting them to cross a road (Guéguen et al., 2015, 2016). Pedestrians behaving in this way could also be more cooperative. The mechanisms involved in maintaining a good reputation can also produce investments to serve the common good (Bshary and Bshary, 2010; Francey and Bergmüller, 2012). Our findings are of practical interest for those designing honesty-based systems, or wishing to maximize contributions to public commodities and services. In a meta-analysis of 15 experiments from 13 research papers (Dear et al., 2019), found a 35\% reduction in the risk of antisocial behavior when eye images are present. In contrast, systematic reviews have suggested that CCTV cameras reduce crime by only $16 \%$. Settling such eyes images nudges on pedestrian signals could have an effect, even a small one, but this could be enough to decrease significantly accidents, particularly considering the group effect of crossings (Pelé et al., 2017). However, how such effect persist in time as pedestrians could get habituated to the eyes as reprimand is absent. However, our study is based on a questionnaire and this nudge needs to be tested in real situations. We encourage authorities to adopt the use of eye image systems in crossing signals in order to decrease the number of illegal crossings and increase pedestrian safety. Field research as well as more ecologically valid situations must be added to laboratory-based studies to show the real effect of these eye images on human cooperative behaviors.

Data availability

Zenodo. Dataset for Eye image effect in the context of pedestrian safety: a French questionnaire study. DOI: https://doi. org/10.5281/zenodo.5745446 
Acknowledgements

We thank Joanna Munro for paper editing service.

References

Alexander RD: The biology of moral systems, The biology of moral systems. Hawthorne, NY, US: Aldine de Gruyter; 1987.

Bateson M, Callow L, Holmes JR, et al.: Do Images of 'Watching Eyes' Induce Behaviour That Is More Pro-Social or More Normative? A Field Experiment on Littering. PLoS One. 2013; 8(12): e82055.

PubMed Abstract | Publisher Full Text

Bateson M, Nettle D, Roberts G: Cues of being watched enhance cooperation in a real-world setting. Biol. Lett. 2006; 2(3): 412-414. PubMed Abstract | Publisher Full Text

Baxter JS, Macrae C, Manstead AS, et al.: Attributional biases and driver behaviour. Soc. Behav. 1990.

Boyd R, Richerson PJ: Punishment allows the evolution of cooperation (or anything else) in sizable groups. Ethol. Sociobiol. 1992; 13(3): 171-195. Publisher Full Text

Bretz F, Hothorn T, Westfall P: Multiple comparisons using R. CRC Press; 2016.

Bshary A, Bshary R: Self-Serving Punishment of a Common Enemy Creates a Public Good in Reef Fishes. Curr. Biol. 2010; 20(22): 2032-2035. PubMed Abstract | Publisher Full Text

Burnham T, Johnson DDP: The Evolutionary and Biological Logic of Human Cooperation. Anal. Krit. 2005; 27: 113-135.

Publisher Full Text

Coleman JS: Foundations of social theory. Harvard University Press; 1994. De Waal F: Morally Evolved. Primates Philos. Moral. Evolved. 2006: 1-80. Dear K, Dutton K, Fox E: Do 'watching eyes' influence antisocial behavior? A systematic review \& meta-analysis. Evol. Hum. Behav. 2019; 40(3): 269-280.

Publisher Full Text

Deb S, Strawderman L, DuBien J, et al.: Evaluating pedestrian behavior at crosswalks: Validation of a pedestrian behavior questionnaire for the

US population. Accid. Anal. Prev. 2017; 106: 191-201.

PubMed Abstract | Publisher Full Text

Eksler V, Lassarre S, Thomas I: Regional analysis of road mortality in

Europe. Public Health. 2008; 122(9): 826-837.

Publisher Full Text

Eller A, Koschate M, Gilson K: Embarrassment: The ingroup-outgroup audience effect in faux pas situations. Eur. J. Soc. Psychol. 2011; 41(4): 489-500.

Publisher Full Text

Elster J: The cement of society: A survey of social order. Cambridge University Press; 1989.

Emery NJ: The eyes have it: the neuroethology, function and evolution of social gaze. Neurosci. Biobehav. Rev. 2000; 24(6): 581-604.

PubMed Abstract | Publisher Full Text

Engard NC: LimeSurvey. 2009. Visited: Summer 2009.

Reference Source

Ernest-Jones M, Nettle D, Bateson M: Effects of eye images on everyday cooperative behavior: a field experiment. Evol. Hum. Behav. 2011; 32(3): 172-178.

Publisher Full Text

Fehr E, Fischbacher U: Social norms and human cooperation. Trends Cogn. Sci. 2004; 8(4): 185-190.

Publisher Full Text

Fehr E, Gächter S: Altruistic punishment in humans. Nature. 2002; 415(6868): 137-140.

Publisher Full Text

Fehr E, Gächter S: Cooperation and punishment in public goods experiments. Am. Econ. Rev. 2000; 90(4): 980-994.

Publisher Full Text

Fehr E, Schneider F: Eyes are on us, but nobody cares: are eye cues relevant for strong reciprocity?. Proc. R. Soc. B Biol. Sci. 2010; 277(1686): 1315-1323.

Publisher Full Text
Filiz-Ozbay E, Ozbay EY: Effect of an audience in public goods provision. Exp. Econ. 2014; 17(2): 200-214.

Publisher Full Text

Flack JC, Girvan M, de Waal FBM, et al.: Policing stabilizes construction of social niches in primates. Nature. 2006; 439(7075): 426-429.

PubMed Abstract | Publisher Full Text

Flynn C: Woman's Best Friend: Pet Abuse and the Role of Companion Animals in the Lives of Battered Women. Violence Women. 2000; 6(2): 162-177.

Publisher Full Text

Francey D, Bergmüller R: Images of Eyes Enhance Investments in a Real-Life Public Good. PLoS One. 2012; 7: e37397.

PubMed Abstract |Publisher Full Text

Furnham A, McManus C, Scott D: Personality, empathy and attitudes to animal welfare. Anthrozoös. 2003; 16(2): 135-146.

Publisher Full Text

Granié M, Pannetier M, Guého L: Validation française d'une Echelle de Comportements Piétons. Qual. Sécurité Déplacement Piéton Facteurs Enjeux Nouv. Actions. 2011: 289-298.

Granié M-A, Pannetier M, Guého L: Developing a self-reporting method to measure pedestrian behaviors at all ages. Accid. Anal. Prev. 2013; 50: 830-839.

PubMed Abstract | Publisher Full Text

Guéguen N, Eyssartier C, Meineri S: A pedestrian's smile and drivers' behavior: When a smile increases careful driving. J. Saf. Res. 2016; 56 83-88.

PubMed Abstract | Publisher Full Text

Guéguen N, Meineri S, Eyssartier C: A pedestrian's stare and drivers' stopping behavior: A field experiment at the pedestrian crossing. Saf. SCi. 2015; 75: 87-89.

Publisher Full Text

Haidt J: The moral emotions. Handb. Affect. Sci. 2003; 11(2003): 852-870.

Haxby N, Hoffman N, Gobbini N: The distributed human neural system for face perception. Trends Cogn. Sci. 2000; 4(6): 223-233.

Publisher Full Text

Holland C, Hill R: The effect of age, gender and driver status on pedestrians' intentions to cross the road in risky situations. Accid. Anal. Prev. 2007; 39(2): 224-237.

PubMed Abstract | Publisher Full Text

Jayasundara B, Wickramasuriya K, Shakila L: Localisation of the LimeSurvey Software. Presented at the Conference on Localised Systems and Applications (CLSA) 20102010; p. 36.

Jensen K, Vaish A, Schmidt MF: The emergence of human prosociality: aligning with others through feelings, concerns, and norms. Front. Psychol. 2014; 5: 822.

Kellert SR, Berry JK: Attitudes, Knowledge, and Behaviors toward Wildlife as Affected by Gender. Wildl. Soc. Bull. 1973-2006. 1987; 15(3): 363-371.

Keltner D, Anderson C: Saving face for Darwin: The functions and uses of embarrassment. Curr. Dir. Psychol. Sci. 2000; 9(6): 187-192.

Publisher Full Text

Kurzban R, DeScioli P, O'Brien E: Audience effects on moralistic punishment. Evol. Hum. Behav. 2007; 28(2): 75-84.

Publisher Full Text

Lamba S, Mace R: People recognise when they are really anonymous in an economic game. Evol. Hum. Behav. 2010; 31(4): 271-278.

Publisher Full Text

Lassarre $S$, Thomas I: Exploring road mortality ratios in Europe: national versus regional realities. J. R. Stat. Soc. Ser. A Stat. Soc. 2005; 168 : 127-144.

Publisher Full Text

Laurin D, Verreault R, Lindsay J, et al.: Physical Activity and Risk of

Cognitive Impairment and Dementia in Elderly Persons. Arch. Neurol. 
2001; 58(3): 498-504.

Publisher Full Text

Lawton $\mathrm{R}$, Parker $\mathrm{D}$, Manstead AS, et al.: The role of affect in predicting social behaviors: The case of road traffic violations. J. Appl. Soc. Psychol. 1997; 27(14): 1258-1276.

LimeSurvey Project Team, and S: LimeSurvey: An open source survey tool. 2012

Mendes N, Steinbeis N, Bueno-Guerra N, et al.: Preschool children and chimpanzees incur costs to watch punishment of antisocial others. Nat. Hum. Behav. 2018; 2(1): 45-51.

PubMed Abstract | Publisher Full Text

Miller RS: Embarrassment: Poise and peril in everyday life. The Guilford Press; 1996.

Miller SC, Kennedy CC, DeVoe DC, et al.: An Examination of Changes in Oxytocin Levels in Men and Women Before and After Interaction With a Bonded Dog. Anthrozoös. 2009; 22(1): 31-42.

Publisher Full Text

Northover SB, Pedersen WC, Cohen AB, et al.: Artificial surveillance cues do not increase generosity: two meta-analyses. Evol. Hum. Behav. 2017; 38(1): 144-153.

Publisher Full Text

Nugier A, Niedenthal PM, Brauer M, et al.: Moral and angry emotions provoked by informal social control. Cogn. Emot. 2007; 21(8): 1699-1720. Özkan T, Lajunen T: A new addition to DBQ: Positive Driver Behaviours Scale. Transp. Res. Part F Traffic Psychol. Behav. 2005; 8: 355-368. Publisher Full Text

Pelé $\mathrm{M}$, Bellut C, Debergue $\mathrm{E}$, et al.: Cultural influence of social information use in pedestrian road-crossing behaviours. Open $\mathrm{SCi}$. 2017: 4(2): 160739 .

PubMed Abstract | Publisher Full Text

Pelé M, Deneubourg J-L, Sueur C: Decision-Making Processes

Underlying Pedestrian Behaviors at Signalized Crossings: Part 2.

Do Pedestrians Show Cultural Herding Behavior?. Safety. 2019a; 54: 82.

Publisher Full Text

Pelé M, Deneubourg J-L, Sueur C: Decision-Making Processes

Underlying Pedestrian Behaviors at Signalized Crossing: Part 1.

The First to Step off the Kerb. Safety. 2019b; 5(4): 79.

Publisher Full Text

Penner LA, Dovidio JF, Piliavin JA, et al.: Prosocial behavior: Multilevel perspectives. Annu. Rev. Psychol. 2005; 56: 365-392.

Pfattheicher S, Keller J: The watching eyes phenomenon: The role of a sense of being seen and public self-awareness. Eur. J. Soc. Psychol. 2015; 45(5): 560-566.

Publisher Full Text

Pfeffer K, Hunter $E$ : The effects of peer influence on adolescent pedestrian road-crossing decisions. Traffic Inj. Prev. 2013; 14(4): 434-440. R Development Core Team: $R$ : A language and environment for statistical computing. Vienna, Austria: R Foundation for Statistical Computing; 2009. Rasmussen J: What can be learned from human error reports? Change Work. Life. 1980.
Reason J, Manstead A, Stradling SG, et al.: Errors and violations on the roads: a real distinction?. Ergonomics. 1990; 33(10-11): 1315-1332.

PubMed Abstract | Publisher Full Text

Ren Z, Jiang X, Wang W: Analysis of the influence of pedestrians' eye contact on drivers' comfort boundary during the crossing conflict. Procedia Eng. 2016; 137(2016): 399-406.

Publisher Full Text

Revelle W: An overview of the psych package. Dep. Psychol. Northwest. Univ. 2011; 1-25. Accessed March 32012

Revelle W, Revelle MW: Package 'psych.'. Compr. R Arch. Netw. 2015.

Riedl K, Jensen K, Call J, et al.: No third-party punishment in chimpanzees. Proc. Natl. Acad. Sci. 2012; 109(37): 14824-14829.

PubMed Abstract | Publisher Full Text

Rodriguez Mosquera PM, Uskul AK, Cross SE: The centrality of social image in social psychology. Eur. J. Soc. Psychol. 2011; 41(4): 403-410.

Publisher Full Text

Saeed TU, Burris MW, Labi S, et al.: An empirical discourse on forecasting the use of autonomous vehicles using consumers' preferences. Technol. Forecast. Soc. Change. 2020; 158: 120130.

Publisher Full Text

Sénémeaud C, Sanrey C, Callé N, et al.: The watching-eyes phenomenon and blood donation: Does exposure to pictures of eyes increase blood donation by young adults?. Transfus. Apher. Sci. 2017; 56(2): 168-170.

PubMed Abstract | Publisher Full Text

Sparks A, Barclay P: Eye images increase generosity, but not for long: The limited effect of a false cue. Evol. Hum. Behav. 2013; 34(5): 317-322. Publisher Full Text

Sueur C, Class B, Hamm C, et al.: Different risk thresholds in pedestrian road crossing behaviour: a comparison of French and Japanese approaches. Accid. Anal. Prev. 2013; 58: 59-63.

PubMed Abstract | Publisher Full Text

Sueur C, Piermattéo A, Pelé M: Dataset for Eye image effect in the context of pedestrian safety: a French questionnaire study [Data set] Zenodo. 2021.

Publisher Full Text

Tom A, Granié M-A: Gender differences in pedestrian rule compliance and visual search at signalized and unsignalized crossroads.

Accid. Anal. Prev. 2011; 43(5): 1794-1801.

PubMed Abstract | Publisher Full Tex

Tomasello $\mathrm{M}$, Vaish A: Origins of human cooperation and morality. Annu. Rev. Psychol. 2013; 64: 231-255.

PubMed Abstract | Publisher Full Tex

Yaffe K, Barnes D, Nevitt M, et al.: A Prospective Study of Physical Activity and Cognitive Decline in Elderly Women: Women Who Walk. Arch.

Intern. Med. 2001; 161(14): 1703-1708.

Publisher Full Text

Zuberbühler K: Audience effects. Curr. Biol. 2008; 18(5): R189-R190. Publisher Full Text 


\section{Open Peer Review}

\section{Current Peer Review Status: ?}

\section{Version 1}

Reviewer Report 17 April 2023

https://doi.org/10.5256/f1000research.80016.r167907

(c) 2023 Zhang Y. This is an open access peer review report distributed under the terms of the Creative Commons Attribution License, which permits unrestricted use, distribution, and reproduction in any medium, provided the original work is properly cited.

\section{Yunchang Zhang}

Desay SV, Singapore, Singapore

This is a well-established paper, and I enjoyed reading this paper. Several comments:

1. For the design of experiment, I'm wondering whether eye contact is enough to explain the feeling of friendly, neutral, surprise, anger, etc. I think more rigorous design or evidence is needed here to prove your control and treatment.

2. I felt that a power analysis is missing in this paper, although fruitful statistical analyses have been conducted. A power analysis will be helpful to demonstrate you have enough samples for the following analyses.

3. Section 3.3, the authors should list the procedures of Kaiser-Meyer-Olkin measure and Bartlett's test procedures and explain them in detail in this section. For example, a question will be why does a 0.80 of Kaiser-Meyer-Olkin measure is considered satisfactory?

4. When I read Tables 2 and 3, I got lost by the meanings of variables. What are the values of these variables? Indicators or continuous variables? I recommend using a table for these explanatory variables.

5. Additionally, the authors should state the number of comparisons in the Tukey multiple comparisons and the confidence level you choose to make the adjustments since 2 comparisons and 20 comparisons have significant differences.

6. The discussion of sociodemographic factors on behavior is vague. Neither did the authors provide statistical numbers, nor persuasive explanations about the differences between South of France and the rest of France.

7. I understand that there is limited literature regarding the impact of eye contact on pedestrian behavior, but I expected a well-established paragraph regarding the future directions of your research. For example, is a questionnaire adequate for the study? Would a simulator study or site observations be better etc? 
Is the work clearly and accurately presented and does it cite the current literature? Yes

Is the study design appropriate and is the work technically sound?

Yes

Are sufficient details of methods and analysis provided to allow replication by others?

Yes

If applicable, is the statistical analysis and its interpretation appropriate?

Yes

Are all the source data underlying the results available to ensure full reproducibility? Partly

Are the conclusions drawn adequately supported by the results?

Partly

Competing Interests: No competing interests were disclosed.

Reviewer Expertise: Social Behavior, human interactions.

I confirm that I have read this submission and believe that I have an appropriate level of expertise to confirm that it is of an acceptable scientific standard, however I have significant reservations, as outlined above.

The benefits of publishing with F1000Research:

- Your article is published within days, with no editorial bias

- You can publish traditional articles, null/negative results, case reports, data notes and more

- The peer review process is transparent and collaborative

- Your article is indexed in PubMed after passing peer review

- Dedicated customer support at every stage

For pre-submission enquiries, contact research@f1000.com 